\title{
Public Opinion Mining on Sochi-2014 Olympics
}

\author{
Kirilenko, Andrei ${ }^{\mathrm{a}}$ and Stepchenkova, Svetlana ${ }^{\mathrm{a}}$
}

${ }^{\mathrm{a}}$ Department of Tourism, Recreation \& Sport Management, University of Florida, USA

\begin{abstract}
The requirements of evidence-based policymaking promote interest to realtime monitoring of public's opinions on policy-relevant topics, and social media data mining allows diversification of information portfolio used by public administrators. This study discusses issues in public opinion mining with respect to extraction and analysis of information posted on Twitter about Sochi-2014 Olympic. It focuses on topics discussed on Twitter and sentiment analysis of tweets about the Games. Final database contained 613,333 tweets covering time span from November 1, 2013 until March 31, 2014. Using hash tags the data were classified into the following categories: Anticipation of the Games (9\%), Cheering of the teams (31\%), News (6\%), Events (11\%), Sports (18\%), and Problems \& Politics (15\%). Research reveals considerable differences in the outcomes of machine sentiment classifiers: Deeply Moving, Pattern, and SentiStrength. SentiStrength produced the most suitable results in terms of minimization of incorrectly classified tweets. Methodological implications and directions for future research are discussed.
\end{abstract}

Keywords: Mega-events; Opinion mining; Sentiment analysis; Sochi Olympics; Social media; Twitter. 